\title{
CARACTERIZAÇÃO MORFOLÓGICA DOS ÓRGÃOS INTERNOS DE REPRODUÇÃO DE SPARTOCERA DENTIVENTRIS (BERG, 1884) (HEMIPTERA: COREIDAE) EM DIFERENTES IDADES
}

\author{
S.M. Jahnke ${ }^{1 *}$, L.R. Redaelli1 ${ }^{1 *}$, R.S.S. dos Santos ${ }^{2}$ \\ ${ }^{1}$ Universidade Federal do Rio Grande do Sul, Faculdade de Agronomia, Av. Bento Gonçalves, 7712, CEP \\ 91540-000, Porto Alegre, RS, Brasil. E-mail: mundstock.jahnke@ufrgs.br
}

\section{RESUMO}

\begin{abstract}
Nesse estudo foi descrita, mensurada e ilustrada, a genitália interna de machos e fêmeas de Spartocera dentiventris considerando distintas idades. As alterações mais evidentes no sistema reprodutivo foram observadas na largura e no comprimento dos ovários que permitem a distinção entre os estágios imaturo e reprodutivo. As medidas dos órgãos de reprodução de machos não apresentam diferenças significativas nas diferentes idades.
\end{abstract}

PALAVRAS-CHAVE: Heteroptera, percevejos, reprodução, morfologia.

\section{ABSTRACT}

REPRODUCTIVE ORGANS OF SPARTOCERA DENTIVENTRIS (BERG, 1884) (HEMIPTERA: COREIDAE) AT DIFFERENT AGES. In this work, the internal reproductive organs of Spartocera dentiventris males and females with different ages are described, measured and illustrated. The most significant changes in the reproductive organs were found in the length and width of ovaries, which permit the distinction between immature and reproductive stages. No significant difference was found among the measurements of the reproductive structures of males at the different ages.

KEY WORDS: Heteroptera, bugs, morphology, reproduction.

\section{INTRODUÇÃO}

Spartocera dentiventris (Berg, 1884) (Hemiptera: Coreidae), conhecido como percevejo-cinzento-dofumo, ocasiona reduções quantitativas equalitativas na cultura do fumo, caracterizadas pelo murchamento e enrolamento de folhas novas, em função do hábito alimentar, sugador, desse coreídeo. No Rio Grande do Sul, ocorre em maiores densidades no período de outubro a março nessa cultura (SILVA, 1999), sendo encontrado em plantas hospedeiras alternativas, especialmenteNicotianaalata (Solanaceae), em outras épocas do ano (JAHNKE et al., 2004).

A busca de alternativas para o controle de pragas exige conhecimentos bioecológicos desses insetos, sendo esse uma das principais fontes de subsídios para implementação de táticas de manejo integrado. $\mathrm{O}$ estudo de aspectos relacionados à reprodução da espécie pode subsidiar essa busca, uma vez que atividades reprodutivas, como procura de parceiros, corte e cópula, podem aumentar o risco de predação (MAgnhagen, 1991). Ao realizarem a oviposição, as fêmeas ficam mais suscetíveis à ação de fatores como predação ou medidas de controle em culturas, tais como aplicação de inseticidas de contato.

O conhecimento detalhado dos órgãos internos de reprodução e a avaliação das modificações ocorridas ao longo do desenvolvimento constituem-se em importantes ferramentas para prever tendências sobre estratégias relacionadas ao potencial biótico da espécie, do ciclo de vida, do período de dormência, alterações numéricas, número de gerações e período reprodutivo, (Tyndale-Biscoe, 1984; Redaelli et al., 1995). Entretanto, poucos são os trabalhos que detalham essas estruturas e processos internos em Heteroptera, especialmente em Coreidae. Assim, objetivou-se descrever, ilustrar e mensurar os órgãos internos de reprodução de machos e fêmeas de $S$. dentiventris, comparando indivíduos de diferentes estágios de desenvolvimento reprodutivo.

${ }^{2}$ Embrapa Uva e Vinho, Estação Experimental de Fruticultura Temperada, Vacaria, RS, Brasil.

*UFRGS - Faculdade de Agronomia, Programa de Pós-graduação em Fitotecnia. 


\section{MATERIAL E MÉTODOS}

Insetos adultos coletados em uma lavoura de fumo cultivar Virgínia var. K326, mantida na área experimental do Departamento de Fitossanidade da Faculdade de Agronomia da UFRGS, Porto Alegre, RS, foram levados ao laboratório, mortos em vapores de éter etílico e dissecados em álcool etílico $70 \%$, usando-se microscópio estereoscópio e as imagens feitas com auxílio de câmara clara. Foram realizadas mensurações dos órgãos internos de reprodução, registrando-se a forma e o número de estruturas, a disposição interna e o tamanho para fêmeas imaturas, reprodutivas e pós-reprodutivas. Os insetos emergidos em campo foram marcados utilizando-se caneta de tinta permanente, anotando-se o sexo e a data de emergência. As fêmeas imaturas foram dissecadas dois a três dias após a emergência, enquanto que aquelas em período de oviposição foram coletadas no campo e dissecadas. As pós-reprodutivas foram provenientes de casais mantidos em gaiolas com plantas de fumo na área experimental e dissecadas uma semana após o registro da última oviposição. Mensurações e descrição dos órgãos reprodutivos de machos também foram realizadas, levando-se em conta a idade após a emergência. O primeiro grupo constava de machos coletados e dissecados de um a dois dias após a emergência. No segundo grupo, foram incluídos aqueles com mais de uma semana após a emergência.

Os dados obtidos decada estrutura foram testados quanto à normalidade e a diferença encontrada entre as medidas de insetos imaturos, reprodutivos e pósreprodutivos, comparadas pelo teste Mann-Withney, utilizando-se em todas as análises o programa estatístico Bioestat 5.0 (AYRES, et al., 2005)

\section{RESULTADOS E DISCUSSÃO}

\section{Órgãos reprodutores de fêmeas}

Apresentam um sistema reprodutivo internocomposto por: um par de ovários $(\mathrm{O})$ do tipo meroístico telotrófico com sete ovaríolos (Ov), um par de ovidu- tos laterais (Ol); um oviduto comum curto (Oc); uma câmara genital (CG) e uma espermateca (E) (Fig. 1). Em cada ovários são órgãos pareados, destacandose como as estruturas mais conspícuas do sistema reprodutivo. Em relação ao número de ovaríolos, Pendergrast (1957) salientou que sete é comumente encontrado em Heteroptera. Em cada ovaríolo na porção apical, que se liga ao diafragma, localiza-se o germário, que são ovogônias formadas por células germinativas, seguidas pelo vitelo (SNODGRASS, 1994). Oviteloéconstituído por células reprodutivas misturadas a células de nutrição. Segundo CHAPMAN (1985), em Hemiptera cada ovaríolo possui células germinativas que se dividem para formar grupos de células que permanecem ligadas por pontes citoplasmáticas. Na base dos ovaríolos, junto ao pedicelo percebe-se a presença de oócitos maduros.

Os órgãos reprodutores da fêmea imatura são frágeis, de coloração esbranquiçada e ocupam uma pequena porção da cavidade abdominal. Os ovaríolos são pequenos, coalescentes e apresentam um grande número de traquéolas aderidas (Fig. 1). Nas fêmeas sexualmente maduras os órgãos reprodutivos tornam-se grandemente aumentados, preenchendo praticamente toda cavidade abdominal, os ovaríolos encontram-se repletos de oócitos em diferentes estágios de maturação (Fig. 2). Após o período reprodutivo, os ovários diminuem novamente, pela ausência dos oócitos.

Cada ovaríolo alcança um oviduto lateral longo que, por sua vez, desemboca num pequeno oviduto comum que termina na câmara genital. Segundo SNODGRASS (1994), os Hemiptera apresentam oviduto do tipo acrotrófico que recebe, além dos oócitos, substâncias produzidas por células foliculares. A espermateca apresenta-se como um tubo sem alargamento distal, estreitada na base e com um curto ducto desembocando na parede anterior da câmara genital, o que corrobora a descrição deSCHUH; SLATER (1996), para Coreidae.

Em média, a largura e o comprimento dos ovários nas imaturas é de, aproximadamente, 2,9 e 3 vezes menor, respectivamente, do que nas fêmeas maduras (Tabela 1) (Figs. 1 e 2), não diferindo entre imaturas e pós-reprodutivas.

Tabela 1 - Média $(\mathrm{mm}) \pm$ EP do comprimento e largura dos órgãos reprodutores internos de fêmeas de Spartocera dentiventris em três idades fisiológicas. Imaturas ( $n=20)$; Reprodutivas ( $n=20)$; Pós-reprodutivas ( $n=18)$.

\begin{tabular}{lllllll}
\hline Órgão & \multicolumn{2}{c}{ Imaturas } & \multicolumn{2}{c}{ Reprodutivas } & \multicolumn{2}{c}{ Pós-Reprodutivas } \\
\hline & Comprimento & Largura & Comprimento & Largura & Comprimento & Largura \\
\cline { 2 - 7 } Ovário & $2,34 \pm(0,092) \mathrm{a}$ & $1,56 \pm(0,065) \mathrm{a}$ & $6,95 \pm(0,496) \mathrm{b}$ & $4,52 \pm(0,245) \mathrm{b}$ & $2,86 \pm(0,313) \mathrm{a}$ & $1,55 \pm(0,168) \mathrm{a}$ \\
Ovaríolo & $2,10 \pm(0,089) \mathrm{a}$ & $0,29 \pm(0,018) \mathrm{a}$ & $5,62 \pm(0,486) \mathrm{b}$ & $1,14 \pm(0,035) \mathrm{b}$ & $2,44 \pm(0,265) \mathrm{a}$ & $0,32 \pm(0,053) \mathrm{a}$ \\
Oviduto lateral & $6,07 \pm(0,220) \mathrm{a}$ & $0,43 \pm(0,021) \mathrm{a}$ & $10,99 \pm(1,052) \mathrm{b}$ & $0,68 \pm(0,042) \mathrm{b}$ & $6,43 \pm(0,592) \mathrm{a}$ & $0,53 \pm(0,074) \mathrm{a}$ \\
Oviduto comum & $0,97 \pm(0,083) \mathrm{a}$ & $0,61 \pm(0,037) \mathrm{a}$ & $1,14 \pm(0,168) \mathrm{b}$ & $0,96 \pm(0,065) \mathrm{b}$ & $0,87 \pm(0,048) \mathrm{a}$ & $0,67 \pm(0,052) \mathrm{a}$ \\
Espermateca & $1,18 \pm(0,100) \mathrm{a}$ & $0,43 \pm(0,013) \mathrm{a}$ & $1,12 \pm(0,107) \mathrm{a}$ & $0,56 \pm(0,035) \mathrm{b}$ & $1,16 \pm(0,132) \mathrm{a}$ & $0,48 \pm(0,045) \mathrm{b}$ \\
\hline
\end{tabular}

Valores seguidos da mesma letra na linha, entre as mesmas medidas, não apresentam diferença significativa $(\alpha=0,01)$. 
Tabelas 2 - Média $(\mathrm{mm}) \pm$ EP do comprimento e largura dos órgãos reprodutores internos de machos de Spartocera dentiventris, recém emergidos ( $n=20)$ e com mais de uma semana após a emergência $(n=18)$.

\begin{tabular}{lcccc}
\hline \multirow{2}{*}{ Órgãos } & \multicolumn{2}{c}{ Recém emergidos } & \multicolumn{2}{c}{ Com mais de uma semana } \\
\cline { 2 - 5 } Testículo & Comprimento & Largura & Comprimento & Largura \\
\cline { 2 - 5 } Vaso deferente & $1,65 \pm(0,049)$ & $1,36 \pm(0,073)$ & $1,60 \pm(0,068)$ & $1,45 \pm(0,072)$ \\
Vesícula seminal & $2,46 \pm(0,070)$ & $0,14 \pm(0,022)$ & $2,35 \pm(1,100)$ & $0,11 \pm(0,008)$ \\
Duto ejaculatório & $0,88 \pm(0,029)$ & $0,48 \pm(0,026)$ & $0,92 \pm(0,057)$ & $0,53 \pm(0,035)$ \\
Bulbo ejaculatório & $2,22 \pm(0,112)$ & $0,32 \pm(0,036)$ & $2,18 \pm(0,071)$ & $0,31 \pm(0,020)$ \\
\hline
\end{tabular}

Não houve diferenças significativas entre as médias.

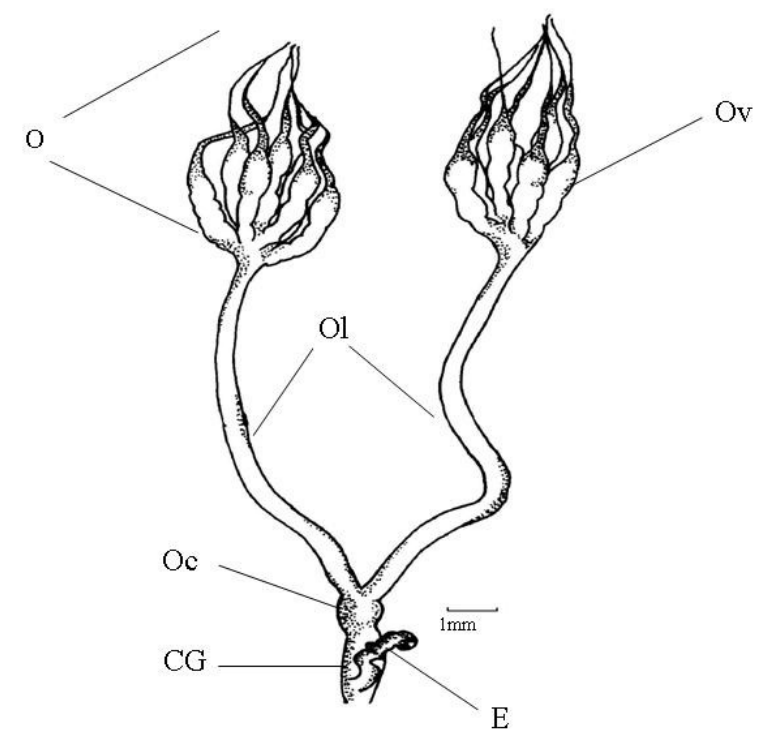

Fig. 1 - Órgãos reprodutores internos de fêmea imatura de Spartocera dentiventris, vista dorsal. Ovários $(\mathrm{O})$; Ovaríolo (Ov); Oviduto comum (Oc); Ovidutos laterais (Ol); Câmara genital (CG); Espermateca (E).

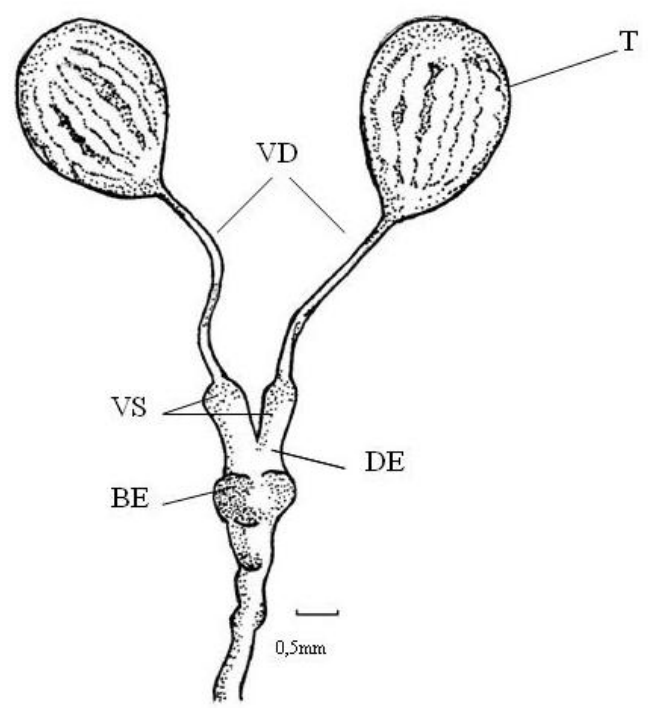

Fig. 3-Órgãos reprodutores internos de macho de Spartocera dentiventris, vista dorsal. Testículos (T); Vaso deferente (VD); Vesículas seminais (VS); Duto ejaculatório (DE); Bulbo ejaculatório (BE).

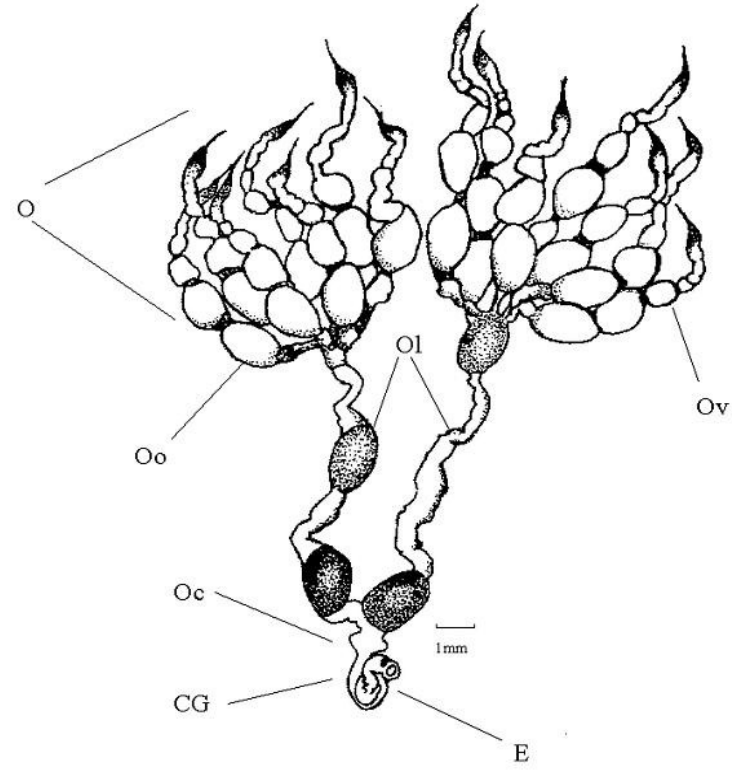

Fig. 2-Órgãos reprodutores internos defêmea sexualmente madura de Spartocera dentiventris, vista dorsal. Ovários $(\mathrm{O})$; Ovaríolo (Ov); Oócito (Oo); Oviduto comum (Oc); Ovidutos laterais (Ol); Câmara genital (CG); Espermateca (E).

A largura dos ovidutos laterais nas fêmeas recém-emergidas é 1,2 vezes menor do que os das reprodutivas. Observou-se que, nas fêmeas em período pós-reprodutivo, os resultados não diferiram em relação às imaturas. $\mathrm{O}$ comprimento do oviduto lateral das fêmeas imaturas é, em média, 1,66 vezes menor do que o das reprodutivas (Tabela 1), possivelmente devido ao acúmulo de oócitos. $\mathrm{O}$ oviduto comum de uma fêmea imatura é 1,61 e 1,52 vezes menor que o de uma fêmea reprodutiva em largura e comprimento, respectivamente. A largura da glândula da espermateca é 1,41 vezes menor e o comprimento da espermateca é 1,15 vezes menor na fêmea imatura. Esses parâmetros indicam que as fêmeas de $S$. dentiventris necessitam de um intervalo de tempo para a maturação de seus órgãos reprodutivos após a emergência. Isso pode ser explicado uma vez que, em muitos insetos, a vitelogênese inicia-se somente após a alimentação da fêmea na fase adulta (SNODGRASS, 1994; CHAPMAN, 1985). Esses resultados corroboram os encontrados por CALDAs et al. (1999) que descreveram a existência de um período de 
pré-oviposição para $S$. dentiventris de 11,8 dias, um reprodutivo de 48,6 dias e longevidade de 66,1 dias. A diminuição no tamanho dos ovários e ovaríolos poderia ser em função da diminuição no número de oócitos presentes. Outro fator que pode influenciar a dimensão desse órgão é o tamanho dos ovos que, segundo PANIZZI (1991), pode variar com a idade das fêmeas em percevejos fitossuccívoros, sendo pequenos no início do período reprodutivo, aumentando com a idade e diminuindo com o envelhecimento.

A longevidade das fêmeas, constatada por CALDAs et al. (1999), ultrapassou o período reprodutivo registrado no presente trabalho, provavelmente por se encontrarem confinadas em gaiolas e protegidas de predação. Entretanto, não se pode afirmar que, em campo, esse padrão se repita, embora existam registros de que fêmeas possam sobreviver de um ano para o outro, conforme observado por Jesus; RomanowsKi (2001). Amaral Filho (1986) também constatou em laboratório uma longevidade de 215,33 dias para fêmeas do coreídeo Crinocerus sanctus (Fabricius, 1770) e considerada mais longa quando comparada com outros heterópteros.

Outro dado que confirma a maior longevidade dessa espécie em campo, são as observações de JAHNKE et al. (2004) de adultos presentes em plantas de $N$. alata, indicando que essa pode permanecer ativa em plantas alterantivas durante os períodos do ano em que não há lavouras comerciais de fumo. Assim, fêmeas consideradas pós-reprodutivas poderiam, mesmo em menor quantidade, vir a colocar ovos viáveis, para garantir a colonização da cultura no ciclo seguinte.

\section{Órgãos reprodutores de machos}

Os machos de S. dentiventris apresentam um par de testículos (T) globoides, situados lateralmente na cavidade abdominal, cada um formado por sete folículos, envolvidos por uma membrana peritoneal de coloração esbranquiçada (Fig. 3) que envolve completamente os testículos e, segundo ImMs (1970), constitui o escroto. Cada testículo continua por um vaso deferente (VD), tubo estreito limitado por epitélio espesso, os quais apresentam, no terço distal, uma região dilatada formando a vesícula seminal (VS). Em alguns Heteroptera pode haver a presença de glândulas acessórias ectademia e mesademia (CHAPMAnN, 1985), embora essas não tenham sido identificadas em $S$. dentiventris. As vesículas seminais abrem-se no duto ejaculatório (DE), o qual apresenta uma dilatação na porção anterior, denominada bulbo ejaculatório (BE). Este duto precede o edeago (Fig.3).

Não foi observada diferença significativa nas medidas dos órgãos reprodutivos daqueles recémemergidos em relação aqueles que apresentavam atividade de cópula (Tabela 2). Embora para muitos coreídeos seja registrado um período de maturação sexual (WANG; MillaR, 2000), não se constatou período pré-reprodutivo nos machos adultos dessa espécie, estando o sistema reprodutivo aparentemente maduro imediatamente após a emergência. Para Leptoglossus clypealis Heidemann, 1910, WANG; Millar (2000) registraram um período de oito dias após a emergência e antes de iniciar o acasalamaneto. Registros de períodos de pré-cópula variando em torno de cinco dias são registrados para Heteroptera (PANIZZI, 1991) que incluem comportamentos específicos. Por outro lado, os machos, estando sexualmente maduros desde a emergência, aumentam a chance de sucesso reprodutivo, por aumentarem as possibilidades de cópulas (HardLing; KaItala, 2004).

No caso dos machos de $S$. dentiventris, não foram registradas características morfométricas que permitissem a diferenciação de um período pré-reprodutivo e para elucidar esse aspecto são necessários exames citológicos (HAYES; WALL ,1999).

\section{CONCLUSÃO}

A avaliação dos órgãos reprodutivos, associada a aspectos biológicos e comportamentais previamente estudados, nos fornece uma ideia da resistência e da adaptação dessa espécie ao ambiente bem como do seu potencial como praga. Da mesma forma, a descrição dos órgãos reprodutivos e das alterações destes em relação aos períodos reprodutivos da espécie trazem importantes acréscimos ao conhecimento da biologia de Coreidae.

\section{AGRADECIMENTOS}

Ao Conselho Nacional de Desenvolvimento Científico e Tecnológico (CNPq) pela bolsa de produtividade concedida ao segundo autor.

\section{REFERÊNCIAS}

AMARAL FILHO, B.F. Observações sobre o ciclo biológico de Crinocerus sanctus (Fabricius, 1775) (Hemiptera: Coreidae) sob condições de laboratório. Anais da Sociedade Entomológica do Brasil, v.15, n.1, p.5-18, 1986.

AYRES, M.; AYRES, M.J.; AYRES, D.L.; SANTOS, A.A.S. Bioest 5.0: aplicações estatísticas nas áreas das ciências biológicas e médicas. Tefé: Mamirauá, 2005. 324p.

CALDAS, B-H.C.; REDAELLI, L.R.; DIEFENBACH, L.M.G. Parâmetros reprodutivos de Corecoris dentiventris Berg (Hemiptera: Coreidae) em cultura de fumo (Nicotiana tabacum). Anais da Sociedade Entomológica do Brasil, v.28, n.4, p.595-600, 1999. 
CHAPMAN, R.F. The insects structure and function. New York: American Elsevier, 1985. 819p.

HARDLING, R.; KAITALA, A.. Male brood care without paternity increases mating success. Behavioral Ecology, v.15, n.5, p.715-721, 2004.

HAYES, E.J.; WALL, R. Age-grading adult insects: a review of techniques. Physiological Entomology, v.24, p.1-10, 1999.

IMMS, A.D. A general textbook of entomology. London: Meuthuen, 1970. 886p.

JAHNKE, S. M.; JESUS, C.R de; ROCHA, L.; REDAELLI, L.R. Registro da ocorrência de Spartocera dentiventris (Berg) (Hem., Coreidae) em Nicotiana alata Link \& Otto (Solanaceae). Acta Biologica Leopoldensia, v.26, n.1, p.151-154, 2004

JESUS, C. R.; ROMANOWSKI, H. P. Population dynamics of Corecoris dentiventris Berg, 1884 (Hemiptera: Coreidae) at an experimental tobacco crop in Porto Alegre, RS - Brazil. Biotemas, v.14, p.47-60, 2001.

MAGNHAGEN, C. Predation risk as a cost of reproduction. Trends of Ecologiy \& Evolution, v.6, p.183-186, 1991.

PANIZZI, A.R. Ecologia Nutricional de insetos sugadores de sementes. In: PANIZZI A.R.; PARRA, J.R.P. (Ed.) Ecologia nutricional de insetos e suas implicações no manejo de pragas. São Paulo: Manole, 1991. p253-287.

PENDERGRAST, J.G. Studies on the reproductive organs of the Heteroptera with a consideration of their bearing on classification. Transactions of the Royal Entomological Society of London, v.109, p.1-63, 1957.

REDAELLI, L.R.; BECKER, M.; ROMANOWSKI, H.P. Changes in the internal reproductive organs and fat body levels as diapause indicators in Oryzophagus oryzae (Coleoptera: Curculionidae). Revista Brasileira de Biologia, v.55, n.4, p.737-744, 1995.

SCHUH, R.T.; SLATER, J.A. True bugs of the world (Hemiptera: Heteroptera): Classification and natural history. New York: Cornell University Press, 1996. 336p.

SILVA, C.R.C. Aspectos da dinâmica populacional e distribuição espacial de Corecoris dentiventris Berg, 1884 (Heteroptera: Coreidae) em cultivo experimental de Nicotiana tabacum (Solanaceae). 1999. 133f. Dissertação (Mestrado em Ecologia) - Departamento de Ecologia, Universidade Federal do Rio Grande do Sul, Porto Alegre, 1999.

SNODGRASS, R.E. Principles of insect morphology. New York: Mc Graw-Hill Book Company, 1994. 667p.

TYNDALE-BISCOE, M. Adaptive significance of brood care of Copris diversus Waterhouse (Coleoptera: Scarabaeidae). Bulletin of Entomological Research, v.74, p.453$461,1984$.

WANG, O.; MILLAR, J. G. Mating Behavior and Evidence for Male-Produced Sex Pheromones in Leptoglossus clypealis (Heteroptera: Coreidae). Annals of The Entomological Society of America, v.93, n.4, p.972-976, 2000.

Recebido em 25/8/09

Aceito em 10/12/10 